\title{
Reconstruction in the Setting of Metastatic Breast Cancer: A Challenging Clinical Question
}

\author{
Jessica Maxwell, MD FRCSC \\ Fred and Pamela Buffett Cancer Center, University of Nebraska Medical Center, Omaha, NE
}

While $12.4 \%$ of women in the US will be diagnosed with breast cancer in their lifetime, a significantly smaller number present with metastatic disease at diagnosis. ${ }^{1}$ It is estimated that approximately $4 \%$ of breast cancer patients present with de novo metastatic disease. ${ }^{2}$ While metastatic breast cancer (MBC) mandates a shift in clinical focus from cure to disease control, overall survival (OS) rates are improving.

Until recently, it has been common practice to offer surgery solely for symptom control and palliation in patients with MBC. Although the use of primary site surgery in $\mathrm{MBC}$ remains controversial, evidence exists supporting prolonged OS in carefully selected patients. ${ }^{3-11}$

Weiss et al. utilized the National Cancer Database (NCDB) to examine the use of reconstruction in MBC patients who underwent mastectomy, and to analyze complication and survival rates. ${ }^{12}$ This is a step further along the continuum of care for stage IV breast cancer patients, and raises an even more controversial question about surgical management. In the setting of increased life expectancy, do quality-of-life concerns merit consideration of breast reconstruction in patients with MBC?

The NCDB database represents more than 1500 Commission on Cancer-accredited facilities and enables assessment of treatment strategies and outcomes for patients with stage IV breast cancer. The authors demonstrated that more than $10 \%$ of patients with MBC have received breast reconstruction surgery, despite minimal evidence to support its safety or effect on patient

\footnotetext{
(C) Society of Surgical Oncology 2018
}

First Received: 27 July 2018;

Published Online: 7 August 2018

J. Maxwell, MD FRCSC

e-mail: jessica.maxwell@unmc.edu satisfaction and quality of life. This strongly suggests a need for data to guide evidence-based care in this small but significant patient group.

The study by Weiss et al. included patients with de novo metastatic disease who underwent unilateral or bilateral mastectomy with or without reconstruction. Overall, $11.5 \%$ of stage IV mastectomy patients underwent reconstruction, with rates increasing significantly by year from 2004 to 2013. Median OS for the reconstruction and non-reconstruction groups was similar, as were 30- and 90-day mortality rates. Reconstruction patients were younger, had fewer metastatic sites, and were more likely to have lowgrade and hormone receptor-positive disease.

Of note, patients who underwent metastasectomy were excluded in order to create a homogenous cohort. It is worth considering that excluded patients who underwent metastasectomy may represent a carefully selected population. Many of the factors that contribute to receipt of metastasectomy are worth considering in reconstruction for MBC patients: young age, oligometastatic disease, less primary disease burden, and longer disease-free intervals. Therefore, by excluding this group, Weiss et al. may have excluded those patients who would be most suitable for more extensive reconstructive surgery in the metastatic setting.

Unplanned readmissions were higher in tissue-based reconstruction than in implant-based surgery, but overall (planned and unplanned) readmissions did not differ across reconstruction type. This differs from previous reports, with Cordeiro et al. demonstrating higher surgical complication rates in metastatic patients using National Surgical Quality Improvement Program (NSQIP) data, albeit for surgery of the primary breast site, not reconstruction. ${ }^{13}$ Complication rate is an important point in MBC patients who often require ongoing systemic treatment for disease control. Low rates of early mortality and readmission seen in this study were cited by the authors to 
ease concerns about delay to systemic therapy post reconstruction. However, outpatient complications are not accurately reflected in these data, and their impact on quality of life and return to treatment are difficult to assess via retrospective NCDB data.

The choice of autologous reconstruction in the metastatic patient also merits mention: this is a larger surgery requiring increased operative and recovery time, with potential for higher rates of complications. For an $\mathrm{MBC}$ patient with increasing but still limited life expectancy, an autologous procedure may not be an obvious choice. In the study by Weiss et al., autologous reconstruction patients were older, with higher rates of $\mathrm{T} 4$ disease. This may reflect the need for extensive resection necessitating autologous closure in this group. This study does not mention staged implant-based reconstruction and use of tissue expanders, which is likely a reflection of the data available through the NCDB. However, timing of implant switch in conjunction with interruption of systemic therapy and consideration of life expectancy in this patient group is an important point for contemplation.

This study highlights important cost issues in the treatment of MBC. In this period of increasing focus on costeffective care, utilization of healthcare dollars in this controversial setting must be carefully balanced with quality-of-life concerns.

Weiss et al. present a thoughtful analysis of trends in reconstruction for women with $\mathrm{MBC}$. This is an important and challenging clinical question for the surgical community, where we must strive to balance patient quality of life with evidence-based care and healthcare utilization burden. Increasing median survival for stage IV breast cancer patients mandates further study on the impact of reconstruction from a multidisciplinary perspective in order to best understand its true risks and benefits.

\section{REFERENCES}

1. SEER Cancer Stat Facts: Female Breast Cancer. Bethesda, MD: National Cancer Institute. https://seer.cancer.gov/statfacts/html/b reast.html. Accessed 13 July 2018.
2. Ries L, Harkins D, Krapcho M, et al. SEER cancer statistics review, 1975-2003. Bethesda, MD: National Cancer Institute. https://seer.cancer.gov/csr/1975-_2003/

3. Babiera GV, Rao R, Feng L, et al. Effect of primary tumor extirpation in breast cancer patients who present with stage IV disease and an intact primary tumor. Ann Surg Oncol. 2006;13(6):776-782.

4. Bafford AC, Burstein HJ, Barkley CR, et al. Breast surgery in stage IV breast cancer: impact of staging and patient selection on overall survival. Breast Cancer Res Treat. 2009;115(1):7-12.

5. Blanchard DK, Shetty PB, Hilsenbeck SG, Elledge RM. Association of surgery with improved survival in stage IV breast cancer patients. Ann Surg. 2008;247(5):732-738.

6. Fields RC, Jeffe DB, Trinkaus K, et al. Surgical resection of the primary tumor is associated with increased long-term survival in patients with stage IV breast cancer after controlling for site of metastasis. Ann Surg Oncol. 2007;14(12):3345-3351.

7. Gnerlich J, Jeffe DB, Deshpande AD, Beers C, Zander C, Margenthaler JA. Surgical removal of the primary tumor increases overall survival in patients with metastatic breast cancer: analysis of the 1988-2003 SEER data. Ann Surg Oncol. 2007;14(8): 2187-2194.

8. Khan SA, Stewart AK, Morrow M. Does aggressive local therapy improve survival in metastatic breast cancer? Surgery. 2002;132(4):620-6 (discussion 626-7).

9. Neuman HB, Morrogh M, Gonen M, Van Zee KJ, Morrow M, King TA. Stage IV breast cancer in the era of targeted therapy: does surgery of the primary tumor matter? Cancer. 2010;116(5):1226-1233.

10. Nguyen DH, Truong PT, Alexander C, et al. Can locoregional treatment of the primary tumor improve outcomes for women with stage IV breast cancer at diagnosis? Int J Radiat Oncol Biol Phys. 2012;84(1):39-45.

11. Ruiterkamp J, Ernst MF, van de Poll-Franse LV, Bosscha K, Tjan-Heijnen VC, Voogd AC. Surgical resection of the primary tumour is associated with improved survival in patients with distant metastatic breast cancer at diagnosis. Eur J Surg Oncol. 2009;35(11):1146-1151.

12. Weiss A, Chu C, Lin H, Shen Y, et al. Reconstruction in the metastatic breast cancer patient-Results from the National Cancer Database. Ann Surg Oncol. 2018. https://doi.org/10.1245/ s10434-018-6693-1.

13. Cordeiro E, Jackson TD, Elhanas A, Cil TD. Higher rate of breast surgery complications in patients with metastatic breast cancer: an analysis of the NSQIP Database. Ann Surg Oncol. 2014;201:3167-3172. 